Archives

18-19 | 1997

Inventaire des archives de Robert Mandrou

\title{
À la redécouverte de la bibliothèque bleue de Troyes
}

\section{Catherine Velay-Vallantin}

\section{Q OpenEdition}

Journals

Édition électronique

URL : http://journals.openedition.org/ccrh/2571

DOI : $10.4000 /$ ccrh. 2571

ISSN : $1760-7906$

Éditeur

Centre de recherches historiques - EHESS

Édition imprimée

Date de publication : 10 octobre 1997

ISSN : 0990-9141

Référence électronique

Catherine Velay-Vallantin, "À la redécouverte de la bibliothèque bleue de Troyes », Les Cahiers du Centre de Recherches Historiques [En ligne], 18-19 | 1997, mis en ligne le 20 février 2009, consulté le 19 avril 2019. URL : http://journals.openedition.org/ccrh/2571; DOI : 10.4000/ccrh.2571

Ce document a été généré automatiquement le 19 avril 2019

Article L.111-1 du Code de la propriété intellectuelle. 


\title{
À la redécouverte de la bibliothèque bleue de Troyes
}

\author{
Catherine Velay-Vallantin
}

1 En octobre 1963, lors d'un colloque tenu à l'Institut d'histoire à Québec, Robert Mandrou présente les premières conclusions de ses recherches sur la Bibliothèque bleue de Troyes : «Littérature de colportage et mentalités paysannes (XVII ${ }^{\mathrm{e}}$ et $\mathrm{xVIII}{ }^{\mathrm{e}}$ siècles) ». Cette communication anticipe la publication de l'ouvrage De la culture populaire aux XVII et XVIII ${ }^{e}$ siècles, paru dans une première édition à Paris chez Stock en 1964, avec pour sous-titre La Bibliothèque bleue de Troyes, puis réédité chez le même éditeur en 1975, reformulé, augmenté d'une nouvelle bibliographie et de nombreux textes en annexe. Cet ouvrage, considéré maintenant comme un classique des études sur le colportage, provoque en 1964 une vive émergence d'intérêts pour ces livrets oubliés depuis quelques décennies par les historiens. Parallèlement et parfois conjointement avec Robert Mandrou, des chercheurs étudient ces éditions bleues selon des angles disciplinaires ou thématiques différents. La «Préface à la nouvelle édition » rend compte de cet engouement: cet ouvrage «n'a pas peu contribué à attirer l'attention des historiens sur les problèmes que pose le devenir des cultures populaires » et «le sujet a suscité plusieurs travaux importants depuis dix ans ». C'est que, longtemps ignorée, la culture populaire a provoqué un regain d'intérêt, en particulier chez les tenants de disciplines connexes à l'histoire, telles que la sociologie et surtout l'histoire de la littérature. La Bibliothèque bleue, en tant que littérature populaire, ou du moins considérée comme telle, n'échappe pas à ce phénomène. Les historiens, de leur côté, y voient un objet culturel digne d'intérêt et donc d'études, essentiellement pour les problèmes d'assignation culturelle que ces éditions soulèvent, mais aussi pour la place originale qu'elles peuvent occuper dans l'histoire du livre. Comment Robert Mandrou a-t-il pu susciter ainsi une telle floraison de travaux? Pourquoi ce "petit livre», selon ses propres termes, a-t-il généré des recherches aujourd'hui aussi novatrices que nombreuses?

2 D'emblée, Robert Mandrou qualifie la Bibliothèque bleue de « populaire ». Il pose ainsi le problème du public de ces livrets et se trouve à l'origine, parmi d'autres auteurs comme 
Mikhail Bakhtine ou encore Marc Soriano, de la distinction, de la séparation entre "culture des élites", "culture savante» ou encore "culture officielle», et "culture populaire ». C'est avant tout en ce sens que l'on a utilisé l'appellation de "populaire» pour qualifier, ou plutôt disqualifier, la Bibliothèque bleue. Dans cette optique, il s'agirait d'une marginalisation et d'une opposition à un ensemble de valeurs admises par l'élite et plus ou moins imposées, à la totalité de la population, par diverses contraintes religieuses, politiques et sociales. Le qualificatif de "populaire » est dès lors attribué à toute forme sociale et culturelle, qualifiée de "marginale » par les tenants de la culture officielle, qui réapparaîtrait dans une occasion particulière et se distinguerait par sa résistance et sa persistance. La sorcellerie, étudiée par Robert Mandrou, serait un des exemples les plus frappants de ces persistances d'anciennes croyances dans le monde rural, malgré les efforts des pouvoirs civils et religieux pour les combattre. En ce sens donc, son étude pourrait fournir des renseignements sur les mentalités, les habitudes, les réactions d'une société en évolution, sous l'influence de diverses contraintes extérieures.

La Bibliothèque bleue offre une autre approche pour obtenir des renseignements comparables. D'un côté, l'investigation de Robert Mandrou est plus redevable à l'histoire sociale qu'à l'« histoire des mentalités »: elle recherche sur le terrain documentaire des informations historiques tangibles, et non pas le socle de représentations idéologiques, souvent fictives, mis en œuvre par les différents groupes concurrents qui exploitent les données culturelles véhiculées par les éditeurs et les colporteurs. Elle trouve son fondement dans les premiers intérêts manifestés par Lucien Febvre à l'égard du colportage, lorsqu'il publie en 1924, dans la Revue de synthèse historique, un compte rendu des travaux de C. Robert-Muller et A. Allix, édités dans la Revue de géographie alpine, sur "Un type d'émigration alpine, les colporteurs de l'Oisans ». Lucien Febvre intègre alors ses réflexions parmi d'autres sur "quelques ouvrages récents de géographie », ce qui a pour effet de légitimer l'analyse sociale du colportage par l'école géographique d'Albert Demangeon. Pourtant, d'une certaine manière, la recherche de Robert Mandrou est à la fois dépendante de cet héritage et novatrice: son intérêt pour la Bibliothèque bleue se veut plus directement ancrée dans l'« histoire totale», une histoire dialectique, "une histoire assumant et reconstituant les interactions qui lient étroitement et inlassablement l'économique, le politique et le culturel, dans le devenir de chaque groupe, de chaque société globale ». C'est alors qu'il intègre à son approche historique une notion nouvelle, celle de représentation culturelle et idéologique, que l'historien se doit de débusquer chez les groupes sociaux et culturels producteurs et récepteurs de livrets bleus. Ainsi l'exprime-t-il en janvier 1972, lorsque, dans un entretien avec Antoine Casanova et François Hincker de La Nouvelle Critique, il revient sur son livre sur la Bibliothèque bleue : « Tout groupe, quel qu'il soit, paysans, grands seigneurs, magistrats, financiers, dans la mesure même où il constitue un groupe cohérent, présentant une certaine homogénéité, se façonne, accepte, assume toute une série de représentations du monde naturel et humain, du Ciel et de la Terre, des rapports sociaux comme des forces surnaturelles. Ces représentations présentent un égal intérêt aux yeux de l'historien. Un des problèmes les plus délicats qu'il doit résoudre est de retrouver les articulations entre les visions du monde, entendues en ce sens, des différents groupes sociaux dans une société à une époque donnée. Il est évident qu'il dispose de moyens plus grands pour reconstituer la vision du monde des magistrats, des financiers; ou pour étudier la vision du monde qui est proposée aux milieux populaires par les couches supérieures; j'en ai donné un exemple à propos de la Bibliothèque bleue, bibliothèque de colportage, aux XVII 
et $\mathrm{XVIII}^{\mathrm{e}}$ siècles. » Cette notion de représentation, explicitée dans ce texte, n'a rien à voir, bien sûr, avec celle mise en avant par Louis Marin. Mais, c'est en essayant de "reconstituer» la vision du monde des diverses communautés en jeu que Robert Mandrou a ouvert la voie à une archéologie des objets et à une histoire des pratiques, dont l'association dynamique a constitué une des bases méthodologiques de l'histoire culturelle. En cernant les livrets de colportage dans leur forme, leur fréquence, leur dispositif, leur structure, puis en tâchant de comprendre les variations historiques de leurs usages, Robert Mandrou utilise bien là les réflexions de Lucien Febvre sur la problématique de l'« outillage mental ». Parallèlement, dans les années cinquante, à partir des représentations mises en scène dans les textes, Norbert Elias dégage tous les dispositifs psychologiques, les modes d'organisation psychique des individus dans leur système de contrôle des affects, identifiés comme autant de constructions historiques. Même si, de nos jours, l'histoire culturelle ne peut pas plus se réduire à une histoire des superstructures dans une perspective marxiste, qu'à une histoire «des» cultures, populaires ou savantes, il reste que Robert Mandrou, participant de ce type de réflexion, et s'opposant à la traditionnelle partition de l'« histoire philosophique » et de l'« histoire antiquaire ", a ouvert la voie à l'histoire critique, scientifique et technique de l'étude du document. En revalorisant le travail interprétatif, il n'a pas souscrit pour autant à l'ancienne histoire philosophique et à sa vision générale du développement, mais a légitimé au contraire une ambition historique, celle de circonscrire son objet.

C'est en historien, mais aussi en héritier lettré, que Robert Mandrou se situe dans la longue lignée savante des érudits qui se sont penchés sur les livrets bleus dès les premières réunions des cabinets de curiosité $d u x^{e}{ }^{e}$ siècle. L'analyse systématique du contenu de la Bibliothèque bleue est pourtant une opération récente. Encore faut-il nuancer cette affirmation, car si le XIX ${ }^{e}$ siècle s'est intéressé de près à cette littérature, au point d'en rééditer un grand nombre d'ouvrages sous des formes diverses, ce sont surtout les romans de chevalerie qui bénéficient de ces éditions savantes. Il n'est qu'à lire l'introduction de Charles Nodier à l'une de ces rééditions pour comprendre dans quelle direction se porte son intérêt : "Tous les hommes [...] ont besoin d'histoires plus ou moins exagérées, qui relèvent la grandeur de leur origine par quelques fables épiques. La Bibliothèque qui se compose de ces merveilleuses traditions écrites est la véritable bibliothèque du peuple. »

$5 \mathrm{Au} \mathrm{XIX}^{\mathrm{e}}$ siècle, une " redécouverte » des romans populaires de la Bibliothèque bleue est donc dans l'air. L'ensemble du contenu de la Bibliothèque bleue ne bénéficie pas cependant du phénomène. Seuls quelques ouvrages, mi-historiques, mi-anthologiques, tentent une analyse plus ou moins systématique du contenu des œuvres. Le premier grand ouvrage consacré à l'examen de cette littérature est celui de Charles Nisard en 1854, l'Histoire des livres populaires ou de la littérature de colportage. Le succès de l'œuvre nécessitera une seconde édition quelques années plus tard. Ainsi en témoigne Nisard dans la préface de cette réédition : «Aujourd'hui que l'étude des livres populaires, étude dont on a bien voulu dire que j'en avais donné le signal et révélé tout l'intérêt, est cultivée avec une sorte d'entraînement, qu'on publie chaque jour des écrits sur cette matière, à Paris, dans les provinces et même à l'étranger, il m'a paru qu'il était opportun de reprendre mon œuvre primitive. » Puis, en 1885, Nisard renouvelle l'opération en publiant son Essai sur le colportage de librairie.

6 Même si Nisard exagère le volume des publications effectuées sur le sujet, il est certain que la littérature populaire est devenue un thème d'étude, essentiellement pour les 
bibliophiles et les historiens locaux : Émile Socard et Louis Morin à Troyes, et plus tard, en 1928, René Hélot à Rouen, collectionneurs attentifs qui s'attachent à revaloriser un corpus que le parti pris disqualifiant de Nisard a condamné. L'habituelle tension entre populisme et misérabilisme qui s'attache à un grand nombre d'études sur les créations populaires se fait ici clairement sentir. Il faut donc attendre la seconde moitié $\mathrm{du} \mathrm{xx}^{\mathrm{e}}$ siècle pour que l'on comprenne tout l'intérêt que l'historien peut tirer de l'étude de ces livrets. Ce sont les ouvrages de Pierre Brochon et de Jean-Pierre Seguin qui donnent une impulsion nouvelle aux études sur le sujet. Ainsi, une bibliographie exhaustive recense pour la période 1970-1981, soit pour onze ans, un total de 84 ouvrages ou articles, alors que la décennie précédente n'en dénombre que 23.

7 L'analyse du contenu de la Bibliothèque bleue est malaisée. Robert Mandrou en est conscient dès ses premières recherches. Trois raisons expliquent cette difficulté. La durée du phénomène tout d'abord: les publications s'étendent sur plus de deux siècles, et l'on est amené à constater, sur une telle période, une certaine évolution du corpus, évolution dont une analyse globale ne peut pas rendre compte. Ensuite, le manque de datation ou des dates fantaisistes rendent difficile la perception de cette évolution. La diffusion par Baudot, au début du XIX $X^{e}$ siècle, d'ouvrages portant une permission accordée à Pierre Garnier, datant du XVIII ${ }^{\mathrm{e}}$ siècle, est un exemple illustrant bien ces problèmes. Le dernier point, et non le moindre, est qu'il faut travailler sur une fraction plus ou moins importante d'un fonds qui semble avoir été beaucoup plus volumineux. C'est ainsi que certains titres, même si leur nombre est limité, ne nous sont jamais parvenus, comme en témoignent les catalogues de livres ou les listes à la fin et sur les couvertures de certains livrets.

8 En ces années soixante, les questionnements que Robert Mandrou fait émerger sont inédits. Que représentent les ouvrages que nous possédons? Sont-ce les meilleures ventes, publiées en grand nombre d'exemplaires et que, par les lois élémentaires de la probabilité, nous avions quelque chance de voir parvenir jusqu'à nous? Mais l'interprétation inverse est, elle aussi, possible ! Les ouvrages à succès sont vendus, lus et relus, et s'abîment donc rapidement, vu leur fragilité naturelle. Ils n'ont donc que bien peu de chances d'être conservés lors de déménagements ou d'héritages. Au contraire, les ouvrages de peu de succès dorment chez l'éditeur et peuvent donc nous parvenir, pourvu qu'un événement extérieur ne disperse pas le stock. Un certain nombre d'ouvrages de la Bibliothèque nationale, pour le xix ${ }^{e}$ siècle surtout, porte l'estampille "Dépôt légal ", ce qui a permis leur conservation, certes, mais ce qui ne nous renseigne pas sur leur succès. Dès son introduction à son livre, Robert Mandrou montre bien qu'il est hasardeux de s'appuyer sur la conservation de quelques exemplaires pour tirer des conclusions sur la popularité de l'ouvrage. Un renseignement plus intéressant semble être la stabilité d'un titre, tout au long de la période retenue, surtout s'il est l'objet de multiples éditions simultanées dans diverses villes. De plus, Robert Mandrou souligne le rôle joué par les livrets à bon marché sortis en énorme quantité durant ces deux siècles de quelques presses champenoises pour être vendus par des colporteurs et il montre comment les éditions parvenues jusqu'à nous constituent, en dépit de la diversité d'origine des textes, un « corpus » révélateur de mentalités et de sensibilités fort différentes des propositions de la littérature savante contemporaine.

Dans un premier temps fort de sa recherche, Robert Mandrou s'interroge sur les raisons d'une telle formule éditoriale. La tradition historiographique attribue à Nicolas Oudot $l^{\prime}$ « invention » de la Bibliothèque bleue au tout début du XVII ${ }^{\mathrm{e}}$ siècle. Son imprimerie, à 
l'enseigne du Chapon d'Or était située à Troyes dans la rue Notre-Dame. Cette opinion sur son origine prévaut au $\mathrm{XIX}^{\mathrm{e}}$ siècle et est rapportée tant par les érudits ayant abordé le sujet, Antoine Le Roux de Lincy ou Charles Nisard, que par les historiens locaux comme Alexandre Assier, A. Socard, Corrard de Bréban, et Louis Morin. Les ouvrages de Robert Mandrou, de Geneviève Bollème et d'Henri-Jean Martin n'innovent pas sur ce point. Cependant, ils posent des problèmes de chronologie, toujours difficiles à vérifier. Et ils démontrent que l'invention éditoriale ne fut pas instantanée. Il n'est, pour s'en convaincre, que de considérer les premiers ouvrages appartenant à ce fonds, en se reportant au catalogue établi par Alfred Morin. Ils ne se singularisent en fait, par rapport aux autres publications de l'époque, que par leur format réduit. En effet ni le papier, ni le nombre de pages, ni même la typographie et l'illustration ne sentent vraiment la recherche du « bon marché » à tout prix. C'est insensiblement que les ouvrages prendront ces caractéristiques. L'intervention menée par Nicolas Oudot consiste donc à rééditer des ouvrages plus anciens, susceptibles de vivre une "seconde jeunesse", pour peu que les conditions économiques de leur conception et de leur distribution soient modifiées, éventuellement après une correction, le plus souvent une réduction du texte. Une telle opération n'est pas le fait du seul Troyen Nicolas Oudot, mais elle a pu avoir lieu à Lyon quelques années auparavant. Pour quelques-uns des titres publiés à Troyes par Nicolas Oudot, il est possible de retrouver une édition antérieure chez certains éditeurs lyonnais, tels Claude Nourry, Pierre de Sainte-Lucie et, surtout, Benoît Rigaud qui, de 1555 à 1597, imprime et « développe à Lyon le commerce des livres à bon marché ». On voit donc que l'attribution de la paternité des bases éditoriales constitutives de la Bibliothèque bleue est délicate. Il est de toute façon certain que parallèlement, ou même antérieurement aux éditions troyennes du début du XVII ${ }^{\mathrm{e}}$ siècle, des ouvrages de même style ont été publiés.

Robert Mandrou analyse le succès qui semble avoir rapidement couronné l'initiative de l'éditeur troyen et de ses successeurs. À cet essor il y a plusieurs explications. Les conditions géographiques tout d'abord: Troyes est située au centre d'une zone, le nordest de la France, limitée par la ligne Saint-Malo-Genève, zone où les taux d'alphabétisation sont les plus élevés, et cela dès le $\mathrm{xvII}^{\mathrm{e}}$ siècle. Les conditions économiques ensuite : l'existence des foires de Champagne, pourtant en sévère déclin à cette époque, n'est pas étrangère au fait que Troyes devienne le centre de la diffusion de cette littérature. Plus vraisemblablement, toutefois, on peut penser qu'une tradition commerciale s'était instituée, puis avait périclité, et que la ville a tenté de la rétablir en recherchant de nouveaux créneaux, en particulier celui de l'édition. De plus, une telle activité a peut-être rencontré à Troyes des conditions favorables à son développement : la crise de l'industrie papetière a pu entraîner la recherche de nouveaux débouchés. La littérature à bon marché en serait donc un. Force est de constater pourtant que le développement de l'imprimerie troyenne n'a pas réussi à rendre à nouveau florissantes les fabriques de papier puisque, à la fin $\mathrm{du} \mathrm{xvII}^{\mathrm{e}}$ siècle, beaucoup disparaissent et que Jacques Oudot est même obligé d'acheter du papier à l'extérieur de la ville.

Quoi qu'il en soit, la prospérité des éditeurs semble être un fait acquis. Robert Mandrou cite deux faits qui appuient cette affirmation. Tout d'abord, le rapide développement de l'impression des livrets bleus. À Troyes même, à la famille des Oudot viennent s'ajouter d'autres imprimeurs concurrents: les Garnier principalement, mais aussi une dizaine d'imprimeurs qui voient là le moyen d'échapper aux difficultés rencontrées dans la profession à cette époque. Et la recette semble bonne, car le même livret peut connaître, en un intervalle de temps restreint, deux éditions concurrentes, sans que le débouché ne 
se ferme. De nouvelles éditions sont même nécessaires quelques années plus tard. Pour illustrer ce fait, l'exemple de l'histoire de Gallien Restauré dont, outre l'édition primitive de Nicolas Oudot datée de 1606, on connait une édition de 1660 chez Nicolas Oudot, son fils, une de 1679 chez Jean Oudot, une de 1709 chez Jacques Oudot, une chez Jean Garnier sans date, une chez Pierre Garnier avec permission de 1728, une du XIX ${ }^{e}$ siècle sans imprimeur ni date. On voit donc, au travers de ces exemples, l'importance d'un marché potentiel qui autorise de telles pratiques sans que la faillite de ses protagonistes ne s'ensuive pour autant.

12 Le second indice de la prospérité des éditeurs est l'importance des biens qu'ils semblent avoir pu acquérir rapidement. Les inventaires, effectués après le décès de certains éditeurs, et dont nous avons connaissance, sont assez éloquents. Robert Mandrou reproduit celui qui suivit la mort de Jacques Oudot en 1772, et qui fait apparaître une fortune de près de 50000 livres. En outre, cet inventaire révèle des stocks qui semblent importants : 3000 douzaines de livrets complets, 3000 rames à plat de brochures déjà imprimées... C'est donc bien d'une véritable industrie qu'il s'agit, du moins au début du XVIII siècle, et à côté des deux grandes familles, les Oudot et les Garnier, on trouve un grand nombre d'autres éditeurs, au succès parfois moins évident, tels les Adenet, Briden, Febvre, Girardon, dont les noms apparaissent plus ou moins fréquemment au bas des couvertures bleues. Il est vraisemblable que seuls quelques-uns sont spécialisés dans la littérature de colportage, et que cette spécialisation n'intervient d'ailleurs que vers la fin du XVII ${ }^{e}$ siècle, quand le succès de la formule est manifeste. En effet, la plupart des imprimeurs-éditeurs conservent d'autres activités, tel C. Briden, qui possède certes, à son actif, quelques ouvrages de colportage, mais surtout qui édite, seul ou en collaboration avec J. Oudot, un grand nombre de catéchismes à l'usage du diocèse de Troyes au tout début du XVIII ${ }^{\mathrm{e}}$ siècle. Ce double débouché pour les imprimeurs de l'époque est d'ailleurs un fait significatif : la littérature "populaire » à bon marché, d'une part; la littérature « officielle » de l'Église, de l'autre. Déjà en 1672, selon Robert Mandrou, Nicolas II Oudot était imprimeur de l'évêque de Troyes. Vers 1700 , c'est également le cas, comme nous l'avons vu, de C. Briden. Plus tard, au cours du xix siècle, de 1820 à 1860 au moins, c'est aussi vrai pour la veuve André. D'abord seule, puis en collaboration avec son gendre André Anner, elle publie de multiples éditions du catéchisme du diocèse de Troyes, ainsi que de nombreux ouvrages édifiants " approuvés par Monseigneur l'Évêque de Troyes ", parallèlement à d'autres ouvrages habituellement contestés par l'Église, comme le classique Miroir d'astrologie naturelle. Ce dualisme, constamment présent, est interprété par Robert Mandrou par la partition étanche des publics, populaire et savant. La circulation possible des éditions, d'un public à l'autre, d'un usage à l'autre, d'une fonction à l'autre, n'est guère envisagée par Robert Mandrou, tant il est convaincu que « les petits livres à couverture bleue, almanachs, contes, cantiques, récits mythiques, ont constitué, dans la réalité, un frein, un obstacle à la prise de conscience des conditions sociales et politiques auxquelles étaient soumis ces milieux populaires ». "Littérature populaire, certes, ajoute-t-il, elle véhicule bien, dans le cas présent, une forme d'aliénation. » Mais, confronté à ce qu'il appelle « la juxtaposition de tendances inconciliables », la prédication morale d'inspiration chrétienne et l'exaltation des passions, Robert Mandrou ouvre par ses interrogations la voie à l'exploitation par les historiens des notions de pratique, de champ, d'habitus, définies par Pierre Bourdieu. Car l'histoire culturelle s'interroge maintenant sur les ruses de l'utilisation, les adaptations, les compromis de la pratique. Elle porte son attention sur le rapport entre les stratégies d'imposition et, comme l'a écrit 
Michel de Certeau, les "tactiques d'appropriation». Roger Chartier, Dominique Julia, Jacques Revel, entre autres, restituent la liberté à l'intérieur des contraintes subies, ils mettent en évidence le jeu des «tactiques d'appropriation». Ils montrent que les individus ne sont jamais complètement façonnés par les modèles qu'on leur impose et combien il est fondamental que l'histoire en tienne compte, au lieu de raisonner en fonction de modèles dominants, la Réforme, la Contre-Réforme, ou encore l'absolutisme. Or, en 1975, dans la nouvelle édition de son livre, Robert Mandrou ajoute un paragraphe à sa conclusion, où il fait part de ses doutes et de ses questionnements: "Cette juxtaposition pose un problème que nous sommes mal armés pour résoudre, attachés que nous sommes par une longue tradition scolaire et ratiocinante à rechercher et identifier les cohérences de comportements et de discours. Sans doute faut-il admettre que ces exigences n'apparaissaient point nécessaires aux rédacteurs des almanachs et autres livrets de colportage. Ou encore que cette juxtaposition relève d'une philosophie des hommes et de la vie plus compréhensive, capable d'admettre en un même individu la cohabitation des contraires. La question doit être laissée ouverte. »

Si Robert Mandrou s'attache en particulier aux éditions du XVIII ${ }^{\mathrm{e}}$ siècle, c'est que c'est une époque faste pour le développement de la littérature de colportage, si l'on en juge par le nombre d'exemplaires conservés et par celui des centres d'édition qui apparaissent en France. Or, paradoxalement, l'édition troyenne semble se stabiliser entre les deux grandes familles des Oudot et des Garnier, ce qui représente une quasi-permanence de quatre éditeurs troyens au minimum. Les autres noms deviennent plus rares sur les couvertures après 1710 . Cette situation dure jusqu'en 1769, date à laquelle le nom des Oudot disparaît. En effet, la fille de Jean IV Oudot, devenue propriétaire du fonds après sa mère Jeanne Royer, le vend à Jean-Antoine Garnier. Pour quelques années, les éditions de colportage se trouvent donc entre les mains de la dynastie des Garnier. Cependant, à la veille de la Révolution, de nouveaux éditeurs apparaissent, tels Jacques Sainton, A.P.F. André. La tourmente révolutionnaire ne semble pas avoir apporté une gêne considérable à l'édition des livrets bleus. Lorque Jean-Antoine Garnier, dernier descendant direct des Garnier, devient fou, sa femme, Gabrielle-Anne Boucherat, prend sa succession. Elle divorce d'ailleurs en 1805 mais continue de publier des ouvrages du fonds Garnier de 1790 à 1830, changeant simplement les couvertures qui portent successivement le nom de "Citoyenne Garnier », puis de « Femme Garnier », et enfin de " $\mathrm{M}^{\text {me }}$ Garnier ».

Une classification des thèmes du corpus était difficile. Robert Mandrou en a réalisé une, sur le seul fonds troyen, soit 450 titres, c'est-à-dire un sondage au dixième. On constate tout d'abord la prédominance des thèmes religieux. Cette prépondérance n'est que le reflet des statistiques concernant les sujets édités à l'époque: les ouvrages religieux étaient généralement prédominants, quel que soit le type de littérature. Encore faudraitil s'interroger sur l'attribution de certains titres à la Bibliothèque bleue du XVII ${ }^{e}$ siècle : "Ouvrages quasi savants, publiés souvent avec dédicace et approbation ecclésiastique, ils sont fortement inspirés de traités abscons de théologie ", selon Robert Mandrou. Une grande partie des ouvrages religieux est cependant constituée de Vies de Saints, de recueils de prières et surtout de Noëls, dont le succès ne se dément pas. La Grande Bible des Noëls est encore éditée par la Veuve André et Anner au milieu du xIX siècle. L'importance relative de la rubrique «information et instruction» vient toutefois contrebalancer la prédominance des thèmes religieux, surtout si l'on y adjoint les almanachs. À tout moment de leur édition, ces almanachs semblent avoir été très demandés aux colporteurs. À l'appui de ces remarques, Robert Mandrou cite le fait que le catalogue de 
Nicolas Oudot comporte treize titres différents dès l'année 1672-1673. Il faut cependant noter que, sous l'apparente diversité des titres, on pouvait trouver un fonds commun appréciable. Ainsi au XIX siècle, Baudot «habille» de couvertures différentes des almanachs identiques, ce qui lui vaut des ennuis avec la commission de censure : «Dans certains pays on ne vend pas un National mais rien que des Babillards, pas un Bavard mais rien que des Radoteurs. Pour ne pas ennuyer la commission, je ne mets, depuis l'estampille, qu'un titre pour les moyens et un titre pour les gros. Une fois ces deux titres et leurs cahiers lus et reçus, j'habille selon les habitudes et idées de chaque pays. »

À côté de ces almanachs on trouve quelques ouvrages scientifiques, traités de médecine, d'arithmétique, et d'autres plus techniques, jardinage, cuisine, soins aux chevaux, mais on rencontre surtout des ouvrages ayant trait aux sciences occultes. Cette rubrique renferme enfin des ouvrages traitant des us et coutumes de certains métiers, boulangers, domestiques, de jeux et divertissements, d'éducation, alphabets pour la première étape, secrétaires fournissant des modèles de lettres, souvent pour déclarer son amour, de conseils, Catéchisme à l'usage des grandes filles pour être mariées. Pour Robert Mandrou, c'est là une mine de renseignements sur les usages des catégories sociales concernées, source qui pourrait être intéressante si elle n'était d'un conformisme déroutant. Comme il le remarque, la littérature de colportage n'est pas "engagée ». Elle n'est de plus guère novatrice dans la description des rapports sociaux.

La rubrique "fiction » se compose de contes de fées, romans de chevalerie, romans, nouvelles, facéties, burlesque et poissard, théâtre, chansons. La mythologie féerique, pour la nommer comme Robert Mandrou, est une des expressions populaires que les siècles suivants ont retenue. Les contes de fées comme Till l'espiègle ou Fortunatus, débordent largement le cadre de cette Bibliothèque bleue et se retrouvent en d'autres lieux et en d'autres temps. Enfin, la catégorie « romans de chevalerie » est mise en avant par Robert Mandrou car c'est surtout à travers elle qu'a été connue la Bibliothèque bleue. Même si son importance dans le fonds troyen est toute relative ( $10 \%$ environ), ces ouvrages ont bénéficié de nombreuses rééditions sous des formes variées. Selon Robert Mandrou, «ce fonds de légendaire historique et épique à la fois constitue un document mythologique d'une exceptionnelle importance, pour saisir ce premier stade de l'histoire vécue comme un récit merveilleux ». Les témoignages allant dans ce sens ne manquent pas. Robert Mandrou cite Lenglet-Dufresnoy, entre autres critiques du XvIII siècle. Tous s'accordent à voir ici ce qui constitue, dans la Bibliothèque bleue, le "genre " par excellence, la "conscience historique populaire». Mais "il serait évidemment sans intérêt de soumettre ces légendes à un examen de critique traditionnelle", nous dit Robert Mandrou. Pourtant, «ce cycle carolingien mérite d'autant plus l'attention qu'il s'est constitué au moment même où l'histoire se renouvelle de façon décisive, abandonnant le style et les limites des vieilles chroniques médiévales : de ce travail, qui a commencé avec les contemporains d'Étienne Pasquier dans la seconde moitié du XvI siècle (avec Fauchet, La Popelinière, du Tillet et bien d'autres) et qui se continue avec les Pithou, de Thou, Jean de Serres, Dupleix, jusqu'à Mézeray, rien n'a été retenu par les libraires troyens. Ignorant ces travaux qui s'appliquaient à réfuter « les fables belles et frivoles » dont l'histoire des chroniques était encombrée, le cycle épique du légendaire carolingien s'est imposé comme la seule évocation acceptée du passé français magnifié avec générosité ». Et l'explication de cette discrimination se trouve, nous dit Robert Mandrou, «dans l'intervention constante du merveilleux ", inhérente à ces récits épiques: "Une bonne part du succès de ces romans a tenu assurément aux prodiges de ces féeries historiques. » 
Il est ici certain que Robert Mandrou manque de peu une analyse sur les discontinuités du discours historique, telle que les historiens ont pu s'y livrer après les apports de Michel Foucault. En étudiant les récits de la Bibliothèque bleue dans leur enchevêtrement avec les textes « historiques", il aurait pu les resituer dans le style qui leur était propre, et démontrer par là combien les fictions des éditeurs s'articulaient à celles des historiens du moment, combien les raisons de la normalité des uns pouvaient croiser celles des autres, combien la structuration rhétorique d'un récit légendaire pouvait se faire l'écho de celle d'un manuel historique, combien enfin les systèmes de représentation de chacun étaient convergents, puisque aussi bien aucun de ces textes n'avait véritablement de valeur documentaire, pas plus Les Quatre Fils Aymon de la Bibliothèque bleue que La Sorcière de Michelet. Et, d'une certaine façon, Robert Mandrou le sait bien lorsque dans son livre Introduction à la France moderne, publié en 1961 et réédité en 1974, il écrit : "Michelet domine de toute sa puissance de rayonnement, allant de cime en cime, de génie en génie, Vésale, Rabelais, Shakespeare, Montaigne, Cervantès, tous réunis autour de ce foyer qu'il a créé de toutes pièces : la Renaissance. » Et il ajoute que s'il s'agit bien d'« une création de toutes pièces", c'est que Michelet envisage l'histoire "comme un rêve idéal et romantique ». Dès lors, les historiens de la culture seront en mesure de concevoir l'histoire comme la «Figurazion" de Norbert Elias, cette manière spécifique d'interdépendance des hommes les uns par rapport aux autres, mais aussi comme une écriture du changement, de la rupture et de la crise, et encore comme une lecture de notre adéquation psychique propre à chaque opération culturelle et sociale. D'édition en édition, la Bibliothèque bleue rend compte de l'inventivité et de l'aventure scripturaires révélées par les Almanachs, les Catéchismes poissards, les Jardins d'amour, les Explications sur les prophéties, la Nouvelle Science des gens de la campagne, le Devoir des savetiers, ou encore les Fables d'Ésope mises en français avec le sens moral et des figures à chaque fable. Mais aucun de ces textes n'aurait pu s'écrire si les historiens des XVIII ${ }^{e}$ et XIX ${ }^{e}$ siècles n'avaient convaincu leurs auteurs et leurs lecteurs que, comme nous le dit George Steiner, « il n'est pour ainsi dire pas de civilisation, pas de conscience individuelle qui ne résonne encore de l'écho d'une lointaine catastrophe » et, puisque « un mauvais tournant a été pris dans «le bois sombre et sacré », l'homme s'est vu obligé de travailler l'existence contre le fil, de peiner socialement et psychologiquement ». 